\title{
THE JOHN-NIRENBERG INEQUALITY WITH SHARP CONSTANTS
}

\author{
ANDREI K. LERNER
}

\begin{abstract}
We consider the one-dimensional John-Nirenberg inequality:

$$
\left|\left\{x \in I_{0}:\left|f(x)-f_{I_{0}}\right|>\alpha\right\}\right| \leq C_{1}\left|I_{0}\right| \exp \left(-\frac{C_{2}}{\|f\|_{*}} \alpha\right) .
$$

A. Korenovskii found that the sharp $C_{2}$ here is $C_{2}=2 / e$. It is shown in this paper that if $C_{2}=2 / e$, then the best possible $C_{1}$ is $C_{1}=\frac{1}{2} e^{4 / e}$.
\end{abstract}

\section{INTRODUCTION}

Let $I_{0} \subset \mathbb{R}$ be an interval and let $f \in L\left(I_{0}\right)$. Given a subinterval $I \subset I_{0}$, set $f_{I}=\frac{1}{|I|} \int_{I} f$ and $\Omega(f ; I)=\frac{1}{|I|} \int_{I}\left|f(x)-f_{I}\right| d x$.

We say that $f \in B M O\left(I_{0}\right)$ if $\|f\|_{*} \equiv \sup _{I \subset I_{0}} \Omega(f ; I)<\infty$. The classical John-Nirenberg inequality [1] says that there are $C_{1}, C_{2}>0$ such that for any $f \in B M O\left(I_{0}\right)$,

$$
\left|\left\{x \in I_{0}:\left|f(x)-f_{I_{0}}\right|>\alpha\right\}\right| \leq C_{1}\left|I_{0}\right| \exp \left(-\frac{C_{2}}{\|f\|_{*}} \alpha\right) \quad(\alpha>0) .
$$

A. Korenovskii [4] (see also [5, p. 77]) found the best possible constant $C_{2}$ in this inequality, namely, he showed that $C_{2}=2 / e$ :

$$
\left|\left\{x \in I_{0}:\left|f(x)-f_{I_{0}}\right|>\alpha\right\}\right| \leq C_{1}\left|I_{0}\right| \exp \left(-\frac{2 / e}{\|f\|_{*}} \alpha\right) \quad(\alpha>0),
$$

and in general the constant $2 / e$ here cannot be increased.

A question about the sharp $C_{1}$ in (1.1) remained open. In [4], (1.1) was proved with $C_{1}=e^{1+2 / e}=5.67323 \ldots$ The method of the proof in [4] was based on the Riesz sunrise lemma and on the use of nonincreasing rearrangements. In this paper we give a different proof of (1.1) yielding the sharp constant $C_{1}=\frac{1}{2} e^{4 / e}=2.17792 \ldots$

2010 Mathematics Subject Classification. 42A05,42B35.

Key words and phrases. BMO, John-Nirenberg inequality, sharp constants. 
Theorem 1.1. Inequality (1.1) holds with $C_{1}=\frac{1}{2} e^{4 / e}$, and this constant is best possible.

We also use as the main tool the Riesz sunrise lemma. But instead of the rearrangement inequalities, we obtain a direct pointwise estimate for any BMO-function (see Theorem 2.2 below). The proof of this result is inspired (and close in spirit) by a recent decomposition of an arbitrary measurable function in terms of mean oscillations (see [2, 6]).

We mention several recent papers 17, 8] where sharp constants in some different John-Nirenberg type estimates were found by means of the Bellman function method.

\section{Proof of Theorem 1.1}

We shall use the following version of the Riesz sunrise lemma [3].

Lemma 2.1. Let $g$ be an integrable function on some interval $I_{0} \subset \mathbb{R}$, and suppose $g_{I_{0}} \leq \alpha$. Then there is at most countable family of pairwise disjoint subintervals $I_{j} \subset I_{0}$ such that $g_{I_{j}}=\alpha$, and $g(x) \leq \alpha$ for almost all $x \in I_{0} \backslash\left(\cup_{j} I_{j}\right)$.

Observe that the family $\left\{I_{j}\right\}$ in Lemma 2.1 may be empty if $g(x)<\alpha$ a.e. on $I_{0}$.

Theorem 2.2. Let $f \in B M O\left(I_{0}\right)$, and let $0<\gamma<1$. Then there is at most countable decreasing sequence of measurable sets $G_{k} \subset I_{0}$ such that $\left|G_{k}\right| \leq \min \left(2 \gamma^{k}, 1\right)\left|I_{0}\right|$ and for a.e. $x \in I_{0}$,

$$
\left|f(x)-f_{I_{0}}\right| \leq \frac{\|f\|_{*}}{2 \gamma} \sum_{k=0}^{\infty} \chi_{G_{k}}(x) .
$$

Proof. Given an interval $I \subseteq I_{0}$, set $E(I)=\left\{x \in I: f(x)>f_{I}\right\}$. Let us show that there is at most countable family of pairwise disjoint subintervals $I_{j} \subset I_{0}$ such that $\sum_{j}\left|I_{j}\right| \leq \gamma\left|I_{0}\right|$ and for a.e. $x \in I_{0}$,

$$
\left(f-f_{I_{0}}\right) \chi_{E\left(I_{0}\right)} \leq \frac{\|f\|_{*}}{2 \gamma} \chi_{E\left(I_{0}\right)}+\sum_{j}\left(f-f_{I_{j}}\right) \chi_{E\left(I_{j}\right)} .
$$

We apply Lemma 2.1 with $g=f-f_{I_{0}}$ and $\alpha=\frac{\|f\|_{*}}{2 \gamma}$. One can assume that $\alpha>0$ and the family of intervals $\left\{I_{j}\right\}$ from Lemma 2.1 is non-empty (since otherwise (2.2) holds trivially only with the first term on the right-hand side). Since $g_{I_{j}}=\alpha$, we obtain

$$
\begin{aligned}
\sum_{j}\left|I_{j}\right|=\frac{1}{\alpha} \int_{\cup_{j} I_{j}}\left(f-f_{I_{0}}\right) d x & \leq \frac{1}{\alpha} \int_{\left\{x \in I_{0}: f(x)>f_{I_{0}}\right\}}\left(f-f_{I_{0}}\right) d x \\
& =\frac{1}{2 \alpha} \Omega\left(f ; I_{0}\right)\left|I_{0}\right| \leq \gamma\left|I_{0}\right| .
\end{aligned}
$$


Further, $f_{I_{j}}=f_{I_{0}}+\alpha$, and hence

$$
f-f_{I_{0}}=\left(f-f_{I_{0}}\right) \chi_{I_{0} \backslash \cup_{j} I_{j}}+\alpha \chi_{\cup_{j} I_{j}}+\sum_{j}\left(f-f_{I_{j}}\right) \chi_{I_{j}} .
$$

This proves (2.2) since $f-f_{I_{0}} \leq \alpha$ a.e. on $I_{0} \backslash \cup_{j} I_{j}$.

The sum on the right-hand side of (2.2) consists of the terms of the same form as the left-hand side. Therefore, one can proceed iterating (2.2). Denote $I_{j}^{1}=I_{j}$, and let $I_{j}^{k}$ be the intervals obtained after the $k$-th step of the process. Iterating (2.2) $m$ times yields

$$
\left(f-f_{I_{0}}\right) \chi_{E\left(I_{0}\right)} \leq \frac{\|f\|_{*}}{2 \gamma} \sum_{k=0}^{m} \sum_{j} \chi_{E\left(I_{j}^{k}\right)}(x)+\sum_{i}\left(f-f_{I_{i}^{m+1}}\right) \chi_{E\left(I_{i}^{m+1}\right)}
$$

(where $I_{j}^{0}=I_{0}$ ). If there is $m$ such that for any $i$ each term of the second sum is bounded trivially by $\frac{\|f\|_{*}}{2 \gamma} \chi_{E\left(I_{i}^{m+1}\right)}$, we stop the process, and we would obtain the finite sum with respect to $k$. Otherwise, let $m \rightarrow \infty$. Using that

$$
\left|\cup_{i} I_{i}^{m+1}\right| \leq \gamma\left|\cup_{l} I_{l}^{m}\right| \leq \cdots \leq \gamma^{m+1}\left|I_{0}\right|,
$$

we get that the support of the second term will tend to a null set. Hence, setting $E_{k}=\cup_{j} E\left(I_{j}^{k}\right)$, for a.e $x \in E\left(I_{0}\right)$ we obtain

$$
\left(f-f_{I_{0}}\right) \chi_{E\left(I_{0}\right)} \leq \frac{\|f\|_{*}}{2 \gamma}\left(\chi_{E\left(I_{0}\right)}(x)+\sum_{k=1}^{\infty} \chi_{E_{k}}(x)\right) .
$$

Observe that $E\left(I_{j}\right)=\left\{x \in I_{j}: f(x)>f_{I_{0}}+\alpha\right\} \subset E\left(I_{0}\right)$. From this and from the above process we easily get that $E_{k+1} \subset E_{k}$. Also, $E_{k} \subset \cup_{j} I_{j}^{k}$, and hence $\left|E_{k}\right| \leq \gamma^{k}\left|I_{0}\right|$.

Setting now $\bar{F}(I)=\left\{x \in I: f(x) \leq f_{I}\right\}$, and applying the same argument to $\left(f_{I_{0}}-f\right) \chi_{F(I)}$, we obtain

$$
\left(f_{I_{0}}-f\right) \chi_{F\left(I_{0}\right)} \leq \frac{\|f\|_{*}}{2 \gamma}\left(\chi_{F\left(I_{0}\right)}(x)+\sum_{k=1}^{\infty} \chi_{F_{k}}(x)\right)
$$

where $F_{k+1} \subset F_{k}$ and $\left|F_{k}\right| \leq \gamma^{k}\left|I_{0}\right|$. Also, $F_{k} \cap E_{k}=\emptyset$. Therefore, summing (2.3) and (2.4) and setting $G_{0}=I_{0}$ and $G_{k}=E_{k} \cup F_{k}, k \geq 1$, we get (2.1).

Proof of Theorem 1.1. Let us show first that the best possible $C_{1}$ in (1.1) satisfies $C_{1} \geq \frac{1}{2} e^{4 / e}$. It suffices to give an example of $f$ on $I_{0}$ such that for any $\varepsilon>0$,

$$
\left|\left\{x \in I_{0}:\left|f(x)-f_{I_{0}}\right|>2(1-\varepsilon)\|f\|_{*}\right\}\right|=\left|I_{0}\right| / 2 .
$$


Let $I_{0}=[0,1]$ and take $f=\chi_{[0,1 / 4]}-\chi_{[3 / 4,1]}$. Then $f_{I_{0}}=0$. Hence, (2.5) would follow from $\|f\|_{*}=1 / 2$. To show the latter fact, take an arbitrary $I \subset I_{0}$. It is easy to see that computations reduce to the following cases: $I$ contains only $1 / 4$ and $I$ contains both $1 / 4$ and $3 / 4$.

Assume that $I=(a, b), 1 / 4 \in I$, and $b<3 / 4$. Let $\alpha=\frac{1}{4}-a$ and $\beta=b-\frac{1}{4}$. Then $f_{I}=\alpha /(\alpha+\beta)$ and

$$
\Omega(f ; I)=\frac{2}{\alpha+\beta} \int_{\left\{x \in I: f>f_{I}\right\}}\left(f-f_{I}\right)=\frac{2 \alpha \beta}{(\alpha+\beta)^{2}} \leq 1 / 2
$$

with $\Omega(f ; I)=1 / 2$ if $\alpha=\beta$.

Consider the second case. Let $I=(a, b), a<1 / 4$ and $b>3 / 4$. Let $\alpha$ be as above and $\beta=b-\frac{3}{4}$. Then

$$
\Omega(f ; I)=\frac{2}{\alpha+\beta+1 / 2} \int_{\left\{x \in I: f>f_{I}\right\}}\left(f-f_{I}\right)=\frac{4 \alpha(4 \beta+1)}{(2 \alpha+2 \beta+1)^{2}} .
$$

Since

$$
\sup _{0 \leq \alpha, \beta \leq 1 / 4} \frac{4 \alpha(4 \beta+1)}{(2 \alpha+2 \beta+1)^{2}}=1 / 2,
$$

this proves that $\|f\|_{*}=1 / 2$. Therefore, $C_{1} \geq \frac{1}{2} e^{4 / e}$. Let us show now the converse inequality.

Let $f \in B M O\left(I_{0}\right)$. Setting $\psi(x)=\sum_{k=0}^{\infty} \chi_{G_{k}}(x)$, where $G_{k}$ are from Theorem 2.2, we have

$$
\begin{aligned}
\left|\left\{x \in I_{0}: \psi(x)>\alpha\right\}\right| & =\sum_{k=0}^{\infty}\left|G_{k}\right| \chi_{[k, k+1)}(\alpha) \\
& \leq\left|I_{0}\right| \sum_{k=0}^{\infty} \min \left(1,2 \gamma^{k}\right) \chi_{[k, k+1)}(\alpha) .
\end{aligned}
$$

Hence, by (2.1),

$$
\begin{aligned}
\left|\left\{x \in I_{0}:\left|f(x)-f_{I_{0}}\right|>\alpha\right\}\right| & \leq\left|\left\{x \in I_{0}: \psi(x)>2 \gamma \alpha /\|f\|_{*}\right\}\right| \\
& \leq\left|I_{0}\right| \sum_{k=0}^{\infty} \min \left(2 \gamma^{k}, 1\right) \chi_{[k, k+1)}\left(2 \gamma \alpha /\|f\|_{*}\right) .
\end{aligned}
$$

This estimate holds for any $0<\gamma<1$. Therefore, taking here the infimum over $0<\gamma<1$, we obtain

$$
\left|\left\{x \in I_{0}:\left|f(x)-f_{I_{0}}\right|>\alpha\right\}\right| \leq \varphi\left(\frac{2 / e}{\|f\|_{*}} \alpha\right)\left|I_{0}\right|,
$$

where

$$
\varphi(\xi)=\inf _{0<\gamma<1} \sum_{k=0}^{\infty} \min \left(2 \gamma^{k}, 1\right) \chi_{[k, k+1)}(\gamma e \xi) .
$$


Thus, the theorem would follow from the following estimate:

$$
\varphi(\xi) \leq \frac{1}{2} e^{\frac{4}{e}-\xi} \quad(\xi>0) .
$$

It is easy to see that $\varphi(\xi)=1$ for $0<\xi \leq 2 / e$, and in this case (2.6) holds trivially. Next, $\varphi(\xi)=\frac{2}{e \xi}$ for $2 / e \leq \xi \leq 4 / e$. Using that the function $e^{\xi} / \xi$ is increasing on $(1, \infty)$ and decreasing on $(0,1)$, we get $\max _{\xi \in[2 / e, 4 / e]} 2 e^{\xi} / e \xi=\frac{1}{2} e^{4 / e}$, verifying (2.6) for $2 / e \leq \xi \leq 4 / e$.

For $\xi \geq 1$ we estimate $\varphi(\xi)$ as follows. Let $\xi \in[m, m+1), m \in \mathbb{N}$. Taking $\gamma_{i}=i / e \xi$ for $i=m$ and $i=m+1$, we get

$$
\begin{aligned}
\varphi(\xi) & \leq 2 \min \left(\left(\frac{m}{e \xi}\right)^{m},\left(\frac{m+1}{e \xi}\right)^{m+1}\right) \\
& =2\left(\left(\frac{m}{e \xi}\right)^{m} \chi_{\left[m, \xi_{m}\right]}(\xi)+\left(\frac{m+1}{e \xi}\right)^{m+1} \chi_{\left[\xi_{m}, m+1\right)}(\xi)\right),
\end{aligned}
$$

where $\xi_{m}=\frac{1}{e} \frac{(m+1)^{m+1}}{m^{m}}$. Using that the function $e^{\xi} / \xi^{m}$ is increasing on $(m, \infty)$ and decreasing on $(0, m)$, by (2.7) we obtain that for $\xi \in$ $[m, m+1)$,

$$
\varphi(\xi) e^{\xi} \leq 2\left(\frac{m}{e \xi_{m}}\right)^{m} e^{\xi_{m}}=2\left(\frac{e^{\frac{1}{e}(1+1 / m)^{m}}}{(1+1 / m)^{m}}\right)^{m+1} \equiv c_{m} .
$$

Let us show now that the sequence $\left\{c_{m}\right\}$ is decreasing. This would finish the proof since $c_{1}=\frac{1}{2} e^{4 / e}$. Let $\eta(x)=(1+1 / x)^{x}$ and $\nu(x)=$ $\left(e^{\eta(x) / e} / \eta(x)\right)^{x+1}$. Then $c_{m}=2 \nu(m)$ and hence it suffices to show that $\nu^{\prime}(x)<0$ for $x \geq 1$. We have

$$
\nu^{\prime}(x)=\nu(x)\left(\log \frac{e}{\eta(x)}-(1-\eta(x) / e) \log (1+1 / x)^{1+x}\right) .
$$

Since $\eta(x)(1+1 / x)>e$, we get $\mu(x)=\frac{\eta(x)}{e-\eta(x)}>x$. From this and from the fact that the function $(1+1 / x)^{1+x}$ is decreasing we obtain

$$
(e / \eta(x))^{\frac{1}{1-\eta(x) / e}}=(1+1 / \mu(x))^{1+\mu(x)}<(1+1 / x)^{1+x},
$$

which is equivalent to that $\nu^{\prime}(x)<0$.

\section{REFERENCES}

[1] F. John and L. Nirenberg, On functions of bounded mean oscillation, Comm. Pure Appl. Math. 14 (1961), 415-426.

[2] T. Hytönen, The $A_{2}$ theorem: remarks and complements, preprint. Available at http://arxiv.org/abs/1212.3840

[3] I. Klemes, A mean oscilation inequality, Proc. Amer. Math. Soc. 93, no. 3, (1985), 497-500. 
[4] A.A. Korenovskii, The connection between mean oscillations and exact exponents of summability of functions, Mat. Sb. 181 (1990), no. 12, 1721-1727 (in Russian); translation in Math. USSR-Sb. 71 (1992), no. 2, 561-567.

[5] A.A. Korenovskii, Mean oscillations and equimeasurable rearrangements of functions. Lect. Notes Unione Mat. Ital., vol. 4, Springer/UMI, Berlin/Bologna, 2007.

[6] A.K. Lerner, A pointwise estimate for local sharp maximal function with applications to singular integrals, Bull. London Math. Soc., 42 (2010), no. 5, 843-856.

[7] L. Slavin and V. Vasyunin, Sharp results in the integral-form John-Nirenberg inequality, Trans. Amer. Math. Soc. 363 (2011), no. 8, 4135-4169.

[8] V. Vasyunin and A. Volberg, Sharp constants in the classical weak form of the John-Nirenberg inequality, preprint. Available at http://arxiv.org/abs/1204.1782

Department of MAthematics, Bar-Ilan University, 5290002 Ramat GAN, ISRAEL

E-mail address: aklerner@netvision.net.il 